\title{
Flatness of families induced by hypersurfaces on flag varieties
}

\author{
Israel Vainsencher 円 $^{\circledR}$ \\ Departamento de Matemática - UFPE
}

\begin{abstract}
We answer a question posed by S. Kleiman concerning flatness of the family of complete quadrics. We also show that any flat family of hypersurfaces on Grassmann varieties induces a flat family of intersections with the corresponding flag variety.
\end{abstract}

\section{Introduction}

Let $\mathbf{S}$ be the variety of complete quadrics, $\mathbf{S}^{\text {nd }}$ the open subset of nondegenerate quadrics and $\mathbf{F}$ the scheme of complete flags in $\mathbb{P}^{n}$. Let $\varphi_{0}: \mathbf{S}^{n d} \rightarrow \mathbf{H i l b}(\mathbf{F})$ be the morphism that assigns to each nondegenerate quadric the locus of its tangent flags. We prove the following.

Theorem. $\varphi_{0}$ extends to a morphism $\varphi: \mathbf{S} \rightarrow \mathbf{H i l b}(\mathbf{F})$.

This answers affirmatively a question S. Kleiman asked in ([K], p.362).

We first show that $\mathbf{S}$ parametrizes a flat family that restricts, over $\mathbf{S}^{\text {nd }}$, to the family of the graphs of the Gauss map (point $\mapsto$ tangent hyperplane) of nondegenerate quadric hypersurfaces. The family pertinent to Kleiman's question is obtained by composing the family of graphs with the appropriate flag bundle (point $\in$ line $\subset \ldots \subset$ hyperplane).

Our proof of flatness for the completed family of graphs relies on Laksov's description [L] of Semple-Tyrrell's "standard" affine open cover of $\mathbf{S}$.

The space of complete conics has recently reappeared as a simple instance of Kontsevich's spaces of stable maps (cf. [P]). It is also instrumental for the counting of rational curves on a K3 surface double cover of the plane (cf. [V]). Complete quadric surfaces play a role in Narasimhan-Trautmann [NT] study of a compactification of a space of instanton bundles.

\footnotetext{
$1 \odot$ Partially supported by Brasil's CNPq. Thanks are due to the UFMG for the warm atmosphere and hospitality during the preparation of this manuscript.
} 
We also show that any flat family of hypersurfaces on Grassmann varieties induces a flat family of subschemes of the corresponding flag variety (cf. (7.2)).

This statement was first obtained as an earlier attempt to answer Kleiman's question. We observe that for the case of quadric hypersurfaces the family described in the proposition does not induce the family of tangent flags. In fact, for conics it yields a double structure on the graph of the Gauss map.(cf. $\$[0$ for details).

\section{The tangent flag to a smooth quadric}

Write $x=\left(x_{1}, \ldots, x_{n+1}\right)$ (resp. $y=\left(y_{1}, \ldots, y_{n+1}\right)$ ) for the vector of homogeneous coordinates in $\mathbb{P}^{n}$ (resp. $\check{\mathbb{P}}^{n}$ ). Let $\mathbf{F}_{0, n-1} \subset \mathbb{P}^{n} \times \check{\mathbb{P}}^{n}$ be the incidence correspondence "point $\in$ hyperplane". It is the zeros of the incidence section $x \cdot y$ of $\mathcal{O}_{\mathbb{P}^{n}}(1) \otimes \mathcal{O}_{\check{\mathbb{P}}^{n}}(1)$.

Let $\kappa \subset \mathbb{P}^{n}$ denote a smooth quadric represented by a symmetric matrix $a$. The Gauss map $\gamma: \kappa \rightarrow \check{\mathbb{P}}^{n}$ is given by $x \mapsto y=x \cdot a$. Hence we have

$$
\gamma^{*}\left(\mathcal{O}_{\check{\mathbb{P}}^{n}}(1)\right)=\mathcal{O}_{\mathbb{P}^{n}}(1) \mid \kappa
$$

The tangent flag $\widetilde{\kappa} \subset \mathbf{F}_{n}$ of $\kappa$ is equal to the restriction of the flag bundle

$$
\mathbf{F}_{n} \rightarrow \mathbf{F}_{0, n-1} \subset \mathbb{P}^{n} \times \check{\mathbb{P}}^{n}
$$

over the graph $\Gamma_{\kappa}$ of $\gamma$. Consequently, flatness of the family $\{\widetilde{\kappa}\}$ of tangent flags is equivalent to flatness of the family of graphs $\left\{\boldsymbol{\Gamma}_{\kappa}\right\}$. The latter will be handled in $\S$.

We proceed to compute the Hilbert polynomial of the graph $\boldsymbol{\Gamma}$ of the Gauss map of a general quadric hypersurface $\kappa \subset \mathbb{P}^{n}$.

1.1 Lemma. Notation as above, the Hilbert polynomial $\chi\left(\mathcal{O}_{\Gamma}\left(\mathcal{L}^{\otimes t}\right)\right)$ with respect to

$$
\mathcal{L}=\left(\mathcal{O}_{\mathbb{P}^{n}}(1) \otimes \mathcal{O}_{\mathbb{P}^{n}}(1)\right)_{\mid \Gamma}
$$

is equal to

$$
\left(\begin{array}{c}
2 t+n \\
n
\end{array}\right)-\left(\begin{array}{c}
2(t-1)+n \\
n
\end{array}\right)
$$


Proof. We have $\mathcal{L} \cong \mathcal{O}_{\mathbb{P}^{n}}(2)_{\mid} \kappa$ under the identification $\Gamma \cong \kappa$. Thus we may compute

$$
\begin{aligned}
\chi\left(\mathcal{L}^{\otimes t}\right) & =\chi\left(\mathcal{O}_{\mathbb{P}^{n}}(2 t)\right)_{\mid \kappa} \\
& =\chi\left(\mathcal{O}_{\mathbb{P}^{n}}(2 t)\right)-\chi\left(\mathcal{O}_{\mathbb{P}^{n}}(2 t-2)\right) \\
& =\left(\begin{array}{c}
2 t+n \\
n
\end{array}\right)-\left(\begin{array}{c}
2(t-1)+n \\
n
\end{array}\right) .
\end{aligned}
$$

\section{Hilbert polynomial of loci of rank 1 matrices}

The image of the Segre imbedding $\mathbb{P}^{n} \times \mathbb{P}^{n} \rightarrow \mathbb{P}^{N}$ is the variety of matrices of rank one. The image $\Delta$ of the diagonal $\mathbb{P}^{n} \rightarrow \mathbb{P}^{n} \times \mathbb{P}^{n} \rightarrow \mathbb{P}^{N}$ is the subvariety of symmetric matrices of rank one. It's Hilbert polynomial is easily found to be

$$
\operatorname{dim}\left(H^{0}\left(\Delta, \mathcal{O}_{\mathbb{P}^{N}}(t)\right)=\left(\begin{array}{c}
2 t+n \\
n
\end{array}\right) .\right.
$$

The bi-homogeneous ideal $I_{\Delta}$ of the diagonal is generated by the $2 \times 2$ minors of the matrix

$$
\left[\begin{array}{cccc}
x_{1} & x_{2} & \ldots & x_{n+1} \\
y_{1} & y_{2} & \ldots & y_{n+1}
\end{array}\right] .
$$

Write

$$
S=k\left[x_{1}, \ldots, x_{n+1}, y_{1}, \ldots, y_{n+1}\right]
$$

for the polynomial ring in $2 n+2$ variables, and let $S_{i, j}$ denote the space of bi-homogeneous polynomials of bi-degree $(i, j)$. We have

$$
\operatorname{dim}_{k} S_{t, t} /\left(I_{\Delta}\right)_{t, t}=\left(\begin{array}{c}
2 t+n \\
n
\end{array}\right) .
$$

Quite generally, for a closed subscheme $X \subseteq \mathbb{P}^{m} \times \mathbb{P}^{n}$ defined by a bi-homogeneous ideal $I \subseteq S$ we have (cf. [KTB], p. 189)

$$
H^{0}\left(X, \mathcal{O}_{\mathbb{P}^{m}}(t) \otimes \mathcal{O}_{\mathbb{P}^{n}}(t)_{\mid X}\right)=S_{t, t} /(I)_{t, t} \quad \text { for all } t>>0
$$

Indeed, the homomorphism

$$
\begin{aligned}
& R=k\left[z_{i, j}\right] /\left\langle z_{i, j} z_{r, s}-z_{r, j} z_{i, s}\right\rangle \longrightarrow S \\
& z_{i, j} \quad \longmapsto x_{i} y_{j}
\end{aligned}
$$


maps $R_{t}$ isomorphically onto $S_{t, t}$. Let the bi-homogeneous ideal $I \subseteq S$ be generated by polynomials of some fixed bidegree $(d, d)$. Its inverse image via (2) generates a homogeneous ideal $I^{\#} \subseteq R$. We have

$$
\left(R / I^{\#}\right)_{t} \simeq S_{t, t} / I_{t, t}
$$

Now for $t>>0$ we may write

$$
\left(R / I^{\#}\right)_{t}=H^{0}\left(X, \mathcal{O}_{\mathbb{P}^{N}}(t)_{\mid X}\right)=H^{0}\left(X, \mathcal{O}_{\mathbb{P}^{m}}(t) \otimes \mathcal{O}_{\mathbb{P}^{n}}(t)_{\mid X}\right)
$$

Let $L(I)$ denote the monomial ideal of initial terms of $I$ with respect to some bigraded monomial order. Then we have the equality of Hilbert functions,

$$
\varphi_{I}(i, j)=\varphi_{L(I)}(i, j)
$$

This is rather standard: let $f_{1}, \ldots, f_{k}$ be linearly independent forms of bidegree $(i, j)$ in $I$. Replacing if needed each $f_{\mu}$ by $f_{\mu}-c f_{\nu}$ for suitable $c \in k$, we may assume their initial terms $L f_{\mu} \neq L f_{\nu}$. Hence the initial terms $L f_{1}, \ldots, L f_{k}$ are linearly independent monomials in $L(I)_{i, j}$. This shows that $\varphi_{I}(i, j) \leq \varphi_{L(I)}(i, j)$. Conversely, pick monomials $g_{1}>\ldots>g_{k}$ in $L(I)_{i, j}$. We have each $g_{\mu}=L f_{\mu}$ for some $f_{\mu} \in I_{i, j}$. It follows that $f_{1}, \ldots, f_{k}$ are linearly independent.

2.1 Lemma. Let $\Gamma_{0}$ be the subscheme of $\mathbb{P}^{n} \times \check{\mathbb{P}}^{n}$ defined by the ideal

$$
\left\langle x_{i} y_{j} \mid 1 \leq i<j \leq n+1\right\rangle+\left\langle\sum x_{i} y_{i}\right\rangle
$$

Then we have

$$
\varphi_{\boldsymbol{\Gamma}_{0}}(t)=\left(\begin{array}{c}
2 t+n \\
n
\end{array}\right)-\left(\begin{array}{c}
2(t-1)+n \\
n
\end{array}\right) .
$$

Proof. The whole point is to notice that the $x_{i} y_{j}$ span the ideal of initial terms of $I_{\Delta}$ with respect to a suitable order. In fact, the set of $2 \times 2$ minors of (1) is known to be a (universal) Gröbner basis for $I_{\Delta}$ (see Sturmfel [BS]). By the above discussion, we may write

$$
\varphi_{L\left(I_{\Delta}\right)}(t)=\varphi_{I_{\Delta}}(t)=\left(\begin{array}{c}
2 t+n \\
n
\end{array}\right) .
$$

\footnotetext{
${ }^{2}$ I'm indebted to P. Gimenez for his precious help on this matter.
} 
One checks at once that $\sum x_{i} y_{i}$ is a nonzero divisor mod $L\left(I_{\Delta}\right)$ (see (7.1) (i)). Therefore

$$
\varphi_{\Gamma_{0}}(t)=\varphi_{L\left(I_{\Delta}\right)}(t)-\varphi_{L\left(I_{\Delta}\right)}(t-1) .
$$

We will deduce flatness for the "completed" family of Gauss maps from the fact that the above Hilbert polynomial at the special point $\boldsymbol{\Gamma}_{0}$ coincides with the generic one (1.1).

\section{Semple-Tyrrell-Laksov cover}

Let $\mathbf{U}_{n}$ denote the group of lower triangular unipotent $(n+1)$-matrices. Thus, $\mathbf{U}_{n}$ is isomorphic to the affine space $\mathbb{A}^{n(n+1) / 2}$ with coordinate functions $u_{i, j}, 1 \leq j \leq$ $i-1, i=2 \ldots n+1$. These are thought of as entries of the matrix,

$$
u=\left[\begin{array}{lllll}
1 & 0 & 0 & \ldots & 0 \\
u_{2,1} & 1 & 0 & \ldots & 0 \\
u_{3,1} & u_{3,2} & 1 & \ldots & 0 \\
\ldots & \ldots & \ldots & \ldots & \ldots \\
u_{n+1,1} & u_{n+1,2} & u_{n+1,3} & u_{n+1, n} & 1
\end{array}\right] .
$$

Let $d_{1}, \ldots, d_{n}$ be coordinate functions in $\mathbb{A}^{n}$. Put

$$
d^{(1)}=\left[\begin{array}{lllll}
1 & 0 & 0 & \cdots & 0 \\
0 & d_{1} & 0 & \cdots & 0 \\
0 & 0 & d_{1} d_{2} & \cdots & 0 \\
\cdots & \cdots & \cdots & \cdots & \cdots \\
0 & 0 & 0 & \cdots & d_{1} d_{2} \cdots d_{n}
\end{array}\right] .
$$

For a matrix $A$ let it's $i$ th adjugate be the matrix $\stackrel{i}{\wedge} A$ of all $i \times i$ minors. We denote by $d^{(i)}$ the matrix obtained from $\wedge^{i} d^{(1)}$ by removing the common factor $d_{1}^{i-1} d_{2}^{i-2} \cdots d_{i-1}$. E.g., for $n=3$ we have

$$
\begin{aligned}
d^{(1)} & =\operatorname{diag}\left(1, d_{1}, d_{1} d_{2}, d_{1} d_{2} d_{3}\right) \\
d^{(2)} & =\operatorname{diag}\left(d_{1}, d_{1} d_{2}, d_{1} d_{2} d_{3}, d_{1}^{2} d_{2}, d_{1}^{2} d_{2} d_{3}, d_{1}^{2} d_{2}^{2} d_{3}\right) /\left(d_{1}\right) \\
& =\operatorname{diag}\left(1, d_{2}, d_{2} d_{3}, d_{1} d_{2}, d_{1} d_{2} d_{3}, d_{1} d_{2}^{2} d_{3}\right) \\
d^{(3)} & =\operatorname{diag}\left(1, d_{3}, d_{2} d_{3}, d_{1} d_{2} d_{3}\right) .
\end{aligned}
$$


The map $\mathbf{U}_{n} \times \mathbb{A}^{n} \rightarrow \mathbf{S} \subset \prod_{i=1}^{i=n} \mathbb{P}\left(S_{2}\left(\bigwedge^{i} k^{n+1 *}\right)\right)$ defined by sending $(u, d)$ to

$$
\left(u d^{(1)} u^{t},(\stackrel{2}{\wedge} u) d^{(2)} \stackrel{2}{\wedge} u^{t}, \ldots,(\stackrel{n}{\wedge} u) d^{(n)} \stackrel{n}{\wedge}^{t} u^{t}\right)
$$

is an isomorphism onto an affine open subset $\mathbf{S}^{0}$ of $\mathbf{S}$. The variety of complete quadrics may be covered by translates of $\mathbf{S}^{0}$ (cf. Laksov $[\mathrm{L}]$ ).

Let $\mathbf{S}_{d}^{0} \cong \mathbf{U}_{n} \times \mathbb{A}_{d}^{n}$ be the principal open piece defined by $d_{1} d_{2} \cdots d_{n} \neq 0$. It maps isomorphically onto an open subvariety of $\mathbf{S}^{\text {nd }}$.

\section{Graph of the Gauss map}

The variety $\mathbf{S}^{n d}$ of nondegenerate quadrics parametrizes a flat family of graphs of Gauss maps. For a nondegenerate quadric represented by a symmetric matrix $a \in \mathbf{S}^{n d}$ the Gauss map is given by $x \mapsto y=x \cdot a$. We define $\mathbb{K}^{n d} \subset \mathbf{S}^{n d} \times \mathbb{P}^{n} \times \check{\mathbb{P}}^{n}$ by the bi-homogeneous ideal generated by the incidence relation $x \cdot y$ together with the $2 \times 2$ minors of the $2 \times(n+1)$ matrix with rows $y, x \cdot z$, where $z$ denotes the generic symmetric matrix. Clearly $\mathbb{K}^{n d} \rightarrow \mathbf{S}^{n d}$ is a map of $\mathbf{G L}_{n+1}-$ homogeneous spaces.

Now write $a=v c^{(1)} v^{t}$ with $v \in \mathbf{U}_{n}, c \in \mathbb{A}_{d}^{n}\left(c^{(1)}\right.$ as in (3)), and put $x^{\prime}=x v$, $y^{\prime}=y\left(v^{-1}\right)^{t}$. We have $y=x a$ iff $y^{\prime}=x^{\prime} c^{(1)}$. Let

$$
\mathbb{K}_{d}^{0} \subset \mathbf{S}_{d}^{0} \times \mathbb{P}^{n} \times \check{\mathbb{P}}^{n}
$$

be defined by $x \cdot y$ together with the $2 \times 2$ minors of the $2 \times(n+1)$ matrix

$$
\left[\begin{array}{ccccc}
x_{1}^{\prime} & d_{1} x_{2}^{\prime} & d_{1} d_{2} x_{3}^{\prime} & \ldots & d_{1} \cdots d_{n} x_{n+1}^{\prime} \\
y_{1}^{\prime} & y_{2}^{\prime} & y_{3}^{\prime} & \ldots & y_{n+1}^{\prime}
\end{array}\right]
$$

where we put $x_{j}^{\prime}=\sum_{i} u_{i j} x_{i}$ and likewise $y_{j}^{\prime}$ denotes the $j$ th entry of $y\left(u^{-1}\right)^{t}$. Thus $\mathbb{K}_{d}^{0}$ is the total space of the family of Gauss maps parametrized by $\mathbf{S}_{d}^{0}$. Note that $\mathbb{K}_{d}^{0} \rightarrow \mathbf{S}_{d}^{0}$ is a smooth quadric bundle. Its fibre over $(I,(1, \ldots, 1)) \in \mathbf{U}_{n} \times \mathbb{A}^{n}{ }_{d}$ is equal to the quadric given by $\sum x_{i}^{2}$ inside the "diagonal" $y_{1}=x_{1}, \ldots, y_{n+1}=x_{n+1}$ of $\mathbb{P}^{n} \times \check{\mathbb{P}}^{n}$.

Let

$$
\mathbb{K}^{0} \subset \mathbf{S}^{0} \times \mathbb{P}^{n} \times \check{\mathbb{P}}^{n}
$$

be defined by $x \cdot y$ together with the ideal

$$
\begin{gathered}
J=\left\langle x_{1}^{\prime} y_{2}^{\prime}-d_{1} y_{1}^{\prime} x_{2}^{\prime}, \ldots, x_{1}^{\prime} y_{n+1}^{\prime}-d_{1} \cdots d_{n} y_{1}^{\prime} x_{n+1}^{\prime},\right. \\
\left.x_{2}^{\prime} y_{3}^{\prime}-d_{2} y_{2}^{\prime} x_{3}^{\prime}, \ldots, x_{n}^{\prime} y_{n+1}^{\prime}-d_{n} y_{n}^{\prime} x_{n+1}^{\prime}\right\rangle
\end{gathered}
$$


obtained by cancelling all $d_{i}$ factors occurring in the above $2 \times 2$ minors. We obviously have $\mathbb{K}_{\mid \mathbf{S}_{d}^{0}}^{0}=\mathbb{K}_{d}^{0}$.

We will show that $\mathbb{K}^{0}$ is the scheme theoretic closure of $\mathbb{K}_{d}^{0}$ in $\mathbf{S}^{0} \times \mathbb{P}^{n} \times \check{\mathbb{P}}^{n}$ (cf. (6.3)).

\section{A torus action}

Notation as in (3), imbed $\mathbb{G}_{m}^{\times n}$ in $\mathbf{G L}_{n+1}$ by sending $c=\left(c_{1}, \ldots, c_{n}\right) \in \mathbb{G}_{m}^{\times n}$ to $c^{(1)}=\operatorname{diag}\left(1, c_{1}, c_{1} c_{2}, \ldots\right)$. We let $\mathbb{G}_{m}^{\times n}$ act on $\mathbf{S}^{0}$ by

$$
c \cdot(v, b)=\left(c^{(1)} v\left(c^{(1)}\right)^{-1},\left(c_{1}^{2} b_{1}, \ldots c_{n}^{2} b_{n}\right)\right) .
$$

This action is compatible with the natural action of $\mathbf{G L}_{n+1}$ on the space $\mathbb{P}\left(S_{2}\left(k^{n+1 *}\right)\right)$ of quadrics, i.e., for a symmetric matrix $a(v, b):=v b^{(1)} v^{t}$ as above, we have

$$
\begin{aligned}
c^{(1)} \cdot a(v, b) & =c^{(1)} a(v, b)\left(c^{(1)}\right)^{t}=c^{(1)} v b^{(1)} v^{t}\left(c^{(1)}\right)^{t} \\
& =c^{(1)} v\left(c^{(1)}\right)^{-1} c^{(1)} b^{(1)} c^{(1)}\left(\left(c^{(1)}\right)^{t}\right)^{-1} v^{t}\left(c^{(1)}\right)^{t} \\
& =c^{(1)} v\left(c^{(1)}\right)^{-1}\left(c^{(1)}\right)^{2} b^{(1)}\left(\left(c^{(1)}\right)^{t}\right)^{-1} v^{t}\left(c^{(1)}\right)^{t} \\
& =a(c \cdot(v, b)) .
\end{aligned}
$$

It can be also easily checked that $\mathbb{G}_{m}^{\times n}$ acts compatibly on $\mathbf{S}^{0} \times \mathbb{P}^{n} \times \check{\mathbb{P}}^{n}$ and $\mathbb{K}^{0}$ is invariant. Indeed, let $((v, b), x, y) \in \mathbb{K}^{0}$. Pick $c \in \mathbb{G}_{m}^{\times n}$. We have

$$
c \cdot((v, b), x, y)=\left(\left(c^{(1)} v\left(c^{(1)}\right)^{-1},\left(c_{1}^{2} b_{1}, \ldots c_{n}^{2} b_{n}\right)\right), x\left(c^{(1)}\right)^{-1}, y\left(c^{(1)}\right)^{t}\right) .
$$

Now $x^{\prime}=x v$ changes to

$$
x^{\prime \prime}=\left(x\left(c^{(1)}\right)^{-1}\right)\left(c^{(1)} v\left(c^{(1)}\right)^{-1}\right)=x v\left(c^{(1)}\right)^{-1}=x^{\prime}\left(c^{(1)}\right)^{-1}
$$

so that the first row $x^{\prime} b^{(1)}$ in (5) (evaluated at $\left.((v, b), x, y)\right)$ changes to

$$
x^{\prime \prime}\left(b^{(1)}\left(c^{(1)}\right)^{2}\right)=x^{\prime}\left(c^{(1)}\right)^{-1}\left(b^{(1)}\left(c^{(1)}\right)^{2}\right)=x^{\prime}\left(b^{(1)} c^{(1)}\right) .
$$

Similarly, $y^{\prime}=y\left(v^{-1}\right)^{t}$ changes to

$$
\left.y^{\prime \prime}=\left(y\left(c^{(1)}\right)^{t}\right)\left(\left(c^{(1)} v\left(c^{(1)}\right)^{-1}\right)^{-1}\right)^{t}=y\left(v^{-1}\right)^{t}\left(c^{(1)}\right)^{t}\right)=y^{\prime} c^{(1)} .
$$

Therefore (5) changes to the matrix with rows $x^{\prime}\left(b^{(1)} c^{(1)}\right)$ and $y^{\prime} c^{(1)}$. Thus evaluation of (7) at $c \cdot((v, b), x, y)$ and at $((v, b), x, y)$ differ only by nonzero multiples. 
5.1 Lemma. The orbit of $(I, 0) \in \mathbf{S}^{0}$ is the unique closed orbit.

Proof. Conjugation of $v \in \mathbf{U}_{n}$ by the diagonal matrix $c^{(1)}$ replaces each entry $v_{i j}, j<i$ by

$$
\begin{aligned}
\left(c^{(1)} v\left(c^{(1)}\right)^{-1}\right)_{i j} & =c_{i i}^{(1)}\left(v\left(c^{(1)}\right)^{-1}\right)_{i j}=c_{i i}^{(1)} v_{i j}\left(\left(c^{(1)}\right)^{-1}\right)_{j j} \\
& =v_{i j} c_{i i}^{(1)} / c_{j j}^{(1)}=v_{i j} c_{i-1} \cdots c_{j} .
\end{aligned}
$$

Thus, letting $c \rightarrow 0$, we see that $(I, 0)$ is in the orbit closure $\overline{\mathbb{G}_{m}^{\times n} \cdot(v, b)}$

\section{Proof of the theorem}

6.1 Lemma. Notation as in (6), the family $\mathbb{I K}^{0} \rightarrow \mathbf{S}^{0}$ is flat.

Proof. Since $\mathbb{K}^{0} \rightarrow \mathbf{S}^{0}$ is equivariant for the $\mathbb{G}_{m}^{\times n}$-action, it suffices to check that the Hilbert polynomial of the fiber over the representative $(I, 0)$ of the unique closed orbit is right, i.e., coincides with the generic one (cf. Hartshorne [H], thm.9.9, p.261). Evaluating (7) at $(I, 0)$ yields the monomial ideal (1). We are done by virtue of (1.1) and (2.1).

6.2 Lemma. Let $f: X \rightarrow Y$ be a flat, surjective morphism of schemes. If $U \subseteq Y$ is open and schematically dense in $Y$ then $f^{-1} U$ is open and schematically dense in $X$.

Proof. We may assume $X, Y$ affine. Let $A \subseteq B$ be a flat ring extension and let $a \in A$ be such that $\operatorname{Spec} A_{a}$ is schematically dense in Spec $A$. This means that every element in $\operatorname{ker}\left(A \rightarrow A_{a}\right)$ is nilpotent. Flatness implies $\operatorname{ker}\left(B \rightarrow B_{a}\right)=\operatorname{ker}\left(A \rightarrow A_{a}\right) \otimes B$. Hence Spec $B_{a}$ is schematically dense in $\operatorname{Spec} B$. 
6.3 Lemma. Notation as in (6) and (41), we have that $\mathbb{K}^{0}$ is equal to the scheme theoretic closure of $\mathbb{K}_{d}^{0}$.

Proof. In view of (6.1), we may apply the previous lemma to $\mathbb{K}^{0} \rightarrow \mathbf{S}^{0} \supset \mathbf{S}_{d}^{0}$.

6.4 Lemma. Let $G$ be an algebraic group and let

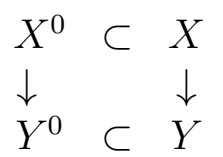

be a commutative diagram of maps of $G$-varieties. Let $\bar{X}, \bar{Y}$ denote the closures of $X^{0}, Y^{0}$. If $\bar{X} \rightarrow \bar{Y}$ is flat over a neighborhood of a point in each closed orbit then $\bar{X} \rightarrow \bar{Y}$ is flat.

Proof. Immediate.

We may now finish the proof of the theorem. Let $\mathbb{K} \subset \mathbf{S} \times \mathbb{P}^{n} \times \check{\mathbb{P}}^{n}$ be the scheme theoretic closure of $\mathbb{K}^{0}$. We have $\mathbb{K} \cap\left(\mathbf{S}^{0} \times \mathbb{P}^{n} \times \check{\mathbb{P}}^{n}\right)=\mathbb{K}^{0}$ flat over $\mathbf{S}^{0}$ by (6.1). The latter is a neighborhood of a point in the unique closed orbit of $\mathbf{S}$. Now apply the previous lemma to $G=\mathbf{G L}_{n+1}, X=\mathbf{S} \times \mathbb{P}^{n} \times \check{\mathbb{P}}^{n}, Y=\mathbf{S}, Y^{0}=\mathbf{S}^{n d}, X^{0}=\mathbb{K}^{n d}$. Finally, since the family of tangent flags is defined by the fibre square,

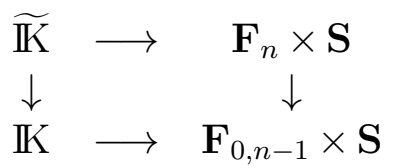

the composition $\widetilde{\mathbb{K}} \rightarrow \mathbb{K} \rightarrow \mathbf{S}$ is flat.

\section{Final remarks}

7.1 (i) The primary decomposition of the monomial ideal in (2.1) can be checked to be given by

$$
\left\langle x_{1}, x_{2}, \ldots, x_{n}\right\rangle \cap \cdots \cap\left\langle x_{1}, \ldots, x_{i}, y_{i+2}, \ldots, y_{n+1}\right\rangle \cap \cdots \cap\left\langle y_{2}, y_{3}, \ldots, y_{n+1}\right\rangle
$$


Thus enlarging it to include the nonzero divisor $x \cdot y$ we see that the special fiber $\boldsymbol{\Gamma}_{0}$ presents no imbedded component.

(ii) The example of $\mathbb{P}^{n}$ acted on by the stabilizer of a point, blown up at that point might clarify why we were not able to show directly that the closure of $\mathbb{K}^{\text {nd }}$ is flat over $\mathbf{S}$.

(iii) For $n=1$ we may write the following global equations for $\mathbb{K}$. Let $z, w$ be a pair of symmetric $3 \times 3$ matrices of independent indeterminates. Then $\mathbb{K} \subset$ $\mathbb{P}^{5} \times \check{\mathbb{P}}^{5} \times \mathbb{P}^{2} \times \check{\mathbb{P}}^{2}$ is given by the $2 \times 2$ minors of the $2 \times 3$ matrices with rows $x \cdot z, y$ and $x, y \cdot w$, in addition to the incidence relation $x \cdot y$ together with the equation $3 z \cdot w=\operatorname{trace}(z \cdot w) I$ for $\mathbf{S} \subset \mathbb{P}^{5} \times \check{\mathbb{P}}^{5}$. It would be nice to give a similar description for higher dimension.

(iv) Still assuming $n=1$, put

$$
\Gamma=\left\{\left(P, \ell, \kappa, \kappa^{\prime}\right) \in \mathbb{P}^{2} \times \check{\mathbb{P}}^{2} \times \mathbb{P}^{5} \times \check{\mathbb{P}}^{5} \mid P \in \kappa \cap \ell, \ell \in \kappa^{\prime}\right\}
$$

It is easy to check that $\boldsymbol{\Gamma}_{\mid \mathbf{S}}=\mathbb{K}$ as sets. Furthermore, $\boldsymbol{\Gamma}$ may be endowed with a natural scheme structure such that $\Gamma \rightarrow \mathbb{P}^{5} \times \check{\mathbb{P}}^{5}$ is flat and with Hilbert polynomial of its fibers equal to $4 t$. Thus, $\boldsymbol{\Gamma}_{\mid \mathbf{S}} \rightarrow \mathbf{S}$ is a family of double structures of genus one on the fibers of $\mathbb{I K}$.

In fact, we have the following.

7.2 Proposition. Any flat family of hypersurfaces on Grassmann varieties induces a flat family of subschemes of the corresponding flag variety.

Before considering the general case, we describe the situation in the projective plane. Thus, let

$$
\mathbf{F}_{2} \subset \mathbb{P}^{2} \times \check{\mathbb{P}}^{2}
$$

be the incidence correspondence "point $\in$ line". Let $f_{0}$ (resp. $f_{1}$ ) denote a curve in $\mathbb{P}^{2}$ (resp. $\left.\check{\mathbb{P}}^{2}\right)$. Set

$$
\Gamma_{\underline{f}}:=\left(f_{0} \times f_{1}\right) \cap \mathbf{F}_{2} .
$$

Then $\boldsymbol{\Gamma}_{\underline{f}}$ is easily seen to be regularly imbedded of codimension 2 in $\mathbf{F}_{2}$ (cf. (7.3)). Moreover, its Hilbert polynomial with respect to the ample sheaf $\mathcal{O}_{\mathbb{P}^{2}}(1) \otimes \mathcal{O}_{\check{\mathbb{P}}^{2}}(1)$ restricted to $\mathbf{F}_{2}$ depends only on the degrees, say $d_{0}, d_{1}$ of $f_{0}, f_{1}$. In fact, the Koszul 
complex that resolves the ideal of $f_{0} \times f_{1}$ in $\mathbb{P}^{2} \times \check{\mathbb{P}}^{2}$ restricts to a resolution of $\boldsymbol{\Gamma}_{\underline{f}}$ in $\mathbf{F}_{2}$. One finds the Hilbert polynomial,

$$
\chi_{\underline{f}}(t)=\left(d_{0}+d_{1}\right) t-d_{0} d_{1}\left(d_{0}+d_{1}-4\right) / 2 .
$$

Therefore, as in the final argument for the proof of (6.1), the parameter space of pairs $\left(f_{0}, f_{1}\right)$, call it $\mathbf{T}\left(=\mathbb{P}^{n_{0}} \times \mathbb{P}^{n_{1}}\right.$ for suitable $\left.n_{0}, n_{1}\right)$, carries a flat family of curves on $\mathbf{F}_{2}$. Precisely, let

$$
\mathbf{W}_{0} \subset \mathbb{P}^{2} \times \mathbb{P}^{n_{0}} \text { and } \mathbf{W}_{1} \subset \check{\mathbb{P}}^{2} \times \mathbb{P}^{n_{1}}
$$

denote the total spaces of the universal plane curve parametrized by $\mathbb{P}^{n_{i}}$. Then

$$
\boldsymbol{\Gamma}:=\left(\mathbf{W}_{0} \underset{\mathbb{P}^{2}}{\times} \mathbf{W}_{1}\right) \bigcap \mathbf{F}_{2} \longrightarrow \mathbf{T}
$$

is a flat family of curves in $\mathbf{F}_{2}$, with fiber $\boldsymbol{\Gamma}_{\underline{f}}$.

For the proof of $(\sqrt{7.2})$ we let $\mathbf{G}_{r, n}$ denote the grassmannian of projective subspaces of dimension $r$ of $\mathbb{P}^{n}$.

Recall that the dimension of the variety of complete flags $\mathbf{F}_{n} \subset \prod \mathbf{G}_{i, n}$ is

$$
\operatorname{dim} \mathbf{F}_{n}=1+\cdots+n
$$

The proposition is an easy consequence of the following.

7.3 Lemma. Let $f_{0}, f_{1}, \ldots, f_{n}$ be arbitrary hypersurfaces of points, lines, ..., hyperplanes in the appropriate grassmannians of subspaces of $\mathbb{P}^{n+1}$. Then the intersection

$$
\boldsymbol{\Gamma}_{\underline{f}}:=\left(f_{0} \times \cdots \times f_{n}\right) \cap \mathbf{F}_{n+1}
$$

is of codimension $n+1$ in $\mathbf{F}_{n+1}$.

Proof. We shall argue by induction on $n$.

We may assume all $f_{i}$ irreducible. Let $n=1$. Pick a line $h \in f_{1}$. Set

$$
h^{(0)}=\left\{P \in \mathbb{P}^{2} \mid P \in h\right\} .
$$

The fiber $\left(\boldsymbol{\Gamma}_{f}\right)_{h} \simeq h^{(0)} \cap f_{0}$ is zero dimensional unless $h^{(0)}=f_{0}$. This occurs for at most one $\bar{h} \in f_{1}$, hence $\Gamma_{\underline{f}}$ is 1-dimensional (otherwise most of its fibres over $f_{1}$ would be at least 1 -dimensional). 
For the inductive step, we set for $h \in \check{\mathbb{P}}^{n+1}$,

$$
h^{(r)}=\left\{g \in \mathbf{G}_{r, n+1} \mid g \subseteq h\right\} \simeq \mathbf{G}_{r, n} .
$$

If the intersection

$$
f_{r}^{\prime}=h^{(r)} \cap f_{r}
$$

were proper for all $r$ and $h \in f_{n}$ then we would be done by induction. Indeed, we have

$$
\left(\boldsymbol{\Gamma}_{\underline{f}}\right)_{h} \simeq\left(f_{0}^{\prime} \times \cdots \times f_{n-1}^{\prime}\right) \cap \mathbf{F}_{n}
$$

By the induction hypothesis, this is of the right dimension

$$
1+\cdots+n-n=1+\cdots+(n-1)
$$

Since $h$ varies in the $n$-dimensional hypersurface $f_{n}$ of $\mathbf{G}_{n, n+1}=\check{\mathbb{P}}^{n+1}$, we would have

$$
\operatorname{dim} \Gamma_{\underline{f}}=(1+\cdots+(n-1))+n=(1+\cdots+(n+1))-(n+1)
$$

as desired.

However, just as in the case $n=1$, it may well happen that the intersection $h^{(r)} \cap f_{r}$ be not proper for some $h, r$. Thus it remains to be shown that, whenever $\operatorname{dim}\left(\boldsymbol{\Gamma}_{\underline{f}}\right)_{h}$ exceeds the right dimension, say by $\delta$, the hyperplane $h$ is restricted to vary in a locus of codimension at least $\delta$ in $f_{n}$. This is taken care of by the lemma below.

7.4 Lemma. Notation as in (9), for $r=0, \ldots, n$ we have

$$
\operatorname{dim}\left\{h \in \check{\mathbb{P}}^{n+1} \mid h^{(r)} \subseteq f_{r}\right\} \leq r .
$$

Proof. Let $\mathbf{F}_{r, n} \subset \check{\mathbb{P}}^{n+1} \times \mathbf{G}_{r, n+1}$ be the partial flag variety. Form the diagram with natural projections,

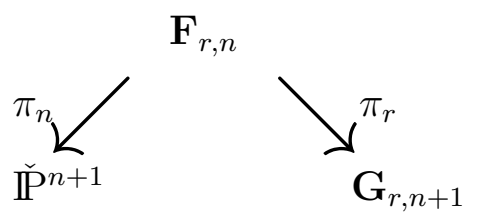

For $g_{r} \in \mathbf{G}_{r, n+1}$, set

$$
g_{r}^{(n)}=\left\{h \in \check{\mathbb{P}}^{n+1} \mid g_{r} \subseteq h\right\} .
$$


We have $g_{r}^{(n)} \simeq \mathbb{P}^{n-r}$ whence it hits any subvariety of $\check{\mathbb{P}}^{n+1}$ of dimension $\geq r+1$. In other words, for any subvariety $\mathbf{Z} \subseteq \check{\mathbb{P}}^{n+1}$ such that $\operatorname{dim} \mathbf{Z} \geq r+1$, we have

$$
\begin{aligned}
\pi_{r} \pi_{n}^{-1} \mathbf{Z} & =\left\{g_{r} \mid \exists h \in \mathbf{Z} \text { s.t. } h \supseteq g_{r}\right\} \\
& =\left\{g_{r} \mid g_{r}^{(n)} \cap \mathbf{Z} \neq \emptyset\right\} \\
& =\mathbf{G}_{r, n+1} .
\end{aligned}
$$

The lemma follows by taking $\mathbf{Z}=\left\{h \in \check{\mathbb{P}}^{n+1} \mid h^{(r)} \subseteq f_{r}\right\}$. Indeed, if $\operatorname{dim} \mathbf{Z} \geq r+1$, then for all $g_{r} \in \mathbf{G}_{r, n+1}$ there exists $h \in \mathbf{Z}$ s.t. $h \supseteq g_{r}$, so $g_{r} \in h^{(r)} \subseteq f_{r}$, contradicting that $f_{r}$ is a hypersurface of $\mathbf{G}_{r, n+1}$.

$\square($ for $(7.4))$

Continuing the proof of (7.3) we consider the stratification of $f_{n}$ by the condition of improper intersection of $f_{r}$ with $h^{(r)}$, namely,

$$
\begin{aligned}
f_{n, 0} & =\left\{h \in f_{n} \mid h^{(0)} \subseteq f_{0}\right\}, \\
f_{n, 1} & =\left\{h \in f_{n} \mid h^{(1)} \subseteq f_{1}\right\} \backslash f_{n, 0}, \\
& \vdots \\
f_{n, n} & =\left\{h \in f_{n} \mid h^{(n)} \subseteq f_{n}\right\} \backslash \bigcup_{j<n} f_{n, j} .
\end{aligned}
$$

We will be done if we show

$$
\operatorname{dim}\left(\boldsymbol{\Gamma}_{\underline{f}}\right)_{h} \leq 1+\cdots+n-r \quad \forall h \in f_{n, r} .
$$

We have already seen that $\operatorname{dim}\left(\boldsymbol{\Gamma}_{\underline{f}}\right)_{h}=1+\cdots+n-1$ for $h$ in $f_{n, n}$. Also, for $r=0$, the desired estimate holds because we have $\left(\boldsymbol{\Gamma}_{\underline{f}}\right)_{h} \subseteq\left(\mathbf{F}_{n+1}\right)_{h} \simeq \mathbf{F}_{n}$ and $\operatorname{dim} \mathbf{F}_{n}=$ $1+\cdots+n$. Let $r>0$ and pick a hyperplane $h \in f_{n, r}$. Then the intersections,

$$
f_{i}^{\prime}=h^{(i)} \cap f_{i},
$$

are proper for $i=0, \ldots, r-1$, whereas for the subsequent index, we have

Thus, we may write,

$$
h^{(r)} \cap f_{r}=h^{(r)} \simeq \mathbf{G}_{r, n} .
$$

$$
\left(\boldsymbol{\Gamma}_{\underline{f}}\right)_{h} \hookrightarrow\left(f_{0}^{\prime} \times \cdots \times f_{r-1}^{\prime} \times \mathbf{G}_{r, n} \times \cdots \times \mathbf{G}_{n-1, n}\right) \bigcap \mathbf{F}_{n} .
$$

By the induction hypothesis the intersection above is of dimension $\operatorname{dim} \mathbf{F}_{n}-r$ in view of the following easy

Remark. The validity of (7.3) for a given $n$ implies properness of the "partial" intersection

$$
\left(f_{0} \times \cdots \times \mathbf{G}_{r, n+1} \times \cdots \times f_{n}\right) \cap \mathbf{F}_{n+1},
$$

where one (or more) of the hypersurfaces $f_{r} \subset \mathbf{G}_{r, n+1}$ is replaced by the corresponding full grassmannian.

$\square($ for $(\overline{7.30})$ (Feb.2'96)

\section{REFERENCES}


[H] R. Hartshorne, Algebraic Geometry, GTM \# 52 Springer-Verlag (1977).

[K] S.L. Kleiman with A. Thorup, "Intersection theory and enumerative geometry: A decade in review", in Algebraic geometry: Bowdoin 1985, S. Bloch, ed., AMS Proc. of Symp. Pure Math, 46-2, p.321-370 (1987).

[KT] S. L. Kleiman \& A. Thorup, Complete bilinear forms, in Algebraic Geometry, Sundance, 1986, eds. A. Holme and R. Speiser, pp. 253-320, Lect. Notes in Math. 1311, Springer-Verlag, Berlin, (1988).

$[\mathrm{KTB}]$ A Geometric Theory of the Buchsbaum-Rim Multiplicity, J. Algebra 167, 168-231 (1994).

[L] D. Laksov Completed quadrics and linear maps, in Algebraic geometry: Bowdoin 1985, S. Bloch, ed., AMS Proc. of Symp. Pure Math., 46-2, p.371-387 (1987).

[NT] M. S. Narasimhan \& G. Trautmann, Compactification of $M_{\mathbb{P}^{3}}(0,2)$ and Poncelet pairs of conics, Pacific J. Math. 145-2, p.255-365 (1990).

[P] R. Pandharipande, “Notes On Kontsevich's Compactification Of The Moduli Space Of Maps, Course notes, Univ. Chicago (1995).

[BS] B. Sturmfels, "Gröbner basis and convex polytopes", Lectures notes at the Holiday Symp. at N. Mexico State Univ., Las Cruces (1994).

[V] I. Vainsencher, "Conics multitangent to a plane curve", in preparation.

Departamento de Matemática

Universidade Federal de Pernambuco

Cidade Universitária 50670-901 Recife-Pe-Brasil

email: israel@dmat.ufpe.br 